\title{
Evolutionarily conserved features of the retained intron in alternative transcripts of the $n x f 1$ (nuclear export factor) genes in different organisms
}

\author{
Ludmila A. Mamon, Sergey F. Kliver, Elena V. Golubkova* \\ Department of Genetics, St. Petersburg State University, St. Petersburg, Russia \\ Email: mamon@LM2010.spb.edu, mahajrod@gmail.com, ${ }^{*}$ gelena@EG10217.spb.edu
}

Received 27 April 2013; revised 30 May 2013; accepted 12 June 2013

Copyright (C) 2013 Ludmila A. Mamon et al. This is an open access article distributed under the Creative Commons Attribution License, which permits unrestricted use, distribution, and reproduction in any medium, provided the original work is properly cited.

\begin{abstract}
One of the features of intron-containing genes of the $n x f$ (nuclear export factor) family in different organisms is the presence of an evolutionarily conserved exon-intron block: exon 110nt-intron-exon 37nt. The intron in this evolutionarily conserved block, which we call a "cassette" intron, can be excised or retained in alternative transcripts of $n x f 1$. It corresponds to intron $10-11$ in the genes that are orthologous to nxf1 in vertebrates, and intron 5 - 6 in the genes that are orthologous to $\mathrm{nxf} 1$ in Drosophilidae. The alignment of sequences of cassette introns in $n x f 1$ genes in vertebrates has revealed four evolutionarily conserved sequences: 1) 5' flanking sequence, 2) a region containing CTE (constitutive transport element), 3) third conserved sequence, and 4) 3' flanking sequence. Introns 5 - 6 of $n x f 1$ in Drosophilidae have no similar conserved sequences. The results of sequence alignment demonstrate a similarity between cassette introns of $n x f 1$ in Drosophilidae in two poly(A) sequences. The prevalence of $D m n x f 1$ transcripts containing cassette intron 5 - 6 under completely spliced transcripts in the heads of adult Drosophila melanogaster suggests a functional importance of transcripts that contain a retained intron. Evolutionary conservation, which in Drosophilidae is evident in the presence of poly(A) sequences in cassette introns of the $n x f 1$ genes, is an adaptive feature: the poly(A) sequences are capable of mimicking the 3'-end of transcripts, promote transport from the nucleus to the cytoplasm, or are involved in NMD control. The ability to form characteristic secondary structures is a common feature of $n x f 1$ cassette introns.
\end{abstract}

Keywords: $N x f$; Intron Retention; CTE; Poly(A);

"Corresponding author.
Drosophila; Vertebrates

\section{INTRODUCTION}

\subsection{The $n x f$ Gene Family and Functions of the nxf1 Gene}

The $n x f$ (nuclear export factor) gene family was named after the function of the universal gene $n x f 1$ responsible for the nuclear-cytoplasmic transport of most mRNAs [1-3]. This path of mRNA export is RanGTP-independent [4]. Several proteins involved in mRNA export pathways are less conserved and presumably appeared later in the evolution of eukaryotes [5]. The blocking of the transport path enabled by the NXF1 protein results in the accumulation of polyadenylated RNAs within the nucleus $[4,6,7]$.

Genes of the $n x f$ family have been found in eukaryotic organisms of the Opisthokonta group, and are characterized by evolutionary conservation [1,5,8]. Genomes of various fungi have only one gene, Mex67, which belongs to this family; animals usually have two to five paralogous genes (see Table 1) [1,9-11]. Plants and some protozoa lack genes of the $n x f$ family [5]. $n x f 1$ genes are the most evolutionarily conserved in the $n x f$ family. What is even more interesting is that $n x f l$ genes across different species of mammals exhibit a much greater degree of similarity than $M m n x f 1$ and $M m n x f 2$ or $H_{s} n x f l$ and $H_{s}$ $n x f 2$ exhibit between themselves (Figure 1).

In the $S$. cerevisiae yeast, the factor Mex67 (mRNA EXport factor of $67 \mathrm{kDa}$ ), which is orthologous to other NXF1 proteins of the eukaryotes of the Opisthokonta group, is also involved in the nuclear-cytoplasmic transport of ribosomal RNAs [5,12,13].

Initially, the NXF1 protein in humans was identified as a potential cytoplasmic cofactor for Tip (tyrosine kinase interacting protein) encoded by the herpesvirus saimiri, and was named TAP (Tip-associated protein) 
Table 1. Characteristics of the genes of the $n x f$ family in different animals: information about the introns retained in alternative transcripts of a number of genes of this family.

\begin{tabular}{|c|c|c|c|c|c|}
\hline Species & $\begin{array}{l}\text { Gene of the } n x f \\
\text { family }\end{array}$ & $\begin{array}{c}\text { Gene localization and } \\
\text { other data }\end{array}$ & Intron number & $\begin{array}{l}\text { Transcript with retained } \\
\text { intron }\end{array}$ & Intron size in nt \\
\hline \multirow{4}{*}{$\begin{array}{c}\text { Drosophila } \\
\text { melanogaster }\end{array}$} & Dm nxf1 & $\mathrm{X}$ & $5-6$ & $+^{*}$ & 1602 \\
\hline & $D m n x f 2$ & $3 \mathrm{~L}$ & none & - & \\
\hline & $D m n x f 3$ & $3 \mathrm{~L}$ & & No introns & \\
\hline & $D m n x f 4$ & $3 R$ & & No introns & \\
\hline Danio rerio & Dr nxf & 21 & $10-11$ & + & 3995 \\
\hline Xenopus tropicalis & $X t n x f 1$ & scaffold_782 & $10-11$ & + & 2596 \\
\hline Monodelphis domestica & $M d n x f 1$ & 5 & $10-11$ & $?$ & 1500 \\
\hline \multirow{4}{*}{ Mus musculus } & $M m n x f 1$ & 19 & $10-11$ & $+{ }^{* *}$ & 1765 \\
\hline & $M m n x f 2$ & $\mathrm{X}$ & $11-12$ & - & 2699 \\
\hline & $M m n x f 3$ & $\mathrm{X}$ & $8-9$ & - & 1423 \\
\hline & $M m n x f 7$ & $\mathrm{X}$ & $11-12$ & + & 1434 \\
\hline \multirow{3}{*}{ Rattus norvegicus } & $\operatorname{Rn} n \times f 1$ & 1 & $10-11$ & + & 1762 \\
\hline & $R n n x f 3$ & $\mathrm{X}$ & $8-9$ & - & 1528 \\
\hline & $R n n x f 7$ & $\mathrm{X}$ & $11-12$ & - & 1424 \\
\hline Canis lupus familiaris & $C l n x f$ & 18 & $10-11$ & $?$ & 1797 \\
\hline Loxodonta africana & La nxfl & & $10-11$ & $?$ & 1810 \\
\hline Equus caballus & Ec $n x f 1$ & 12 & $10-11$ & $?$ & 1815 \\
\hline \multirow{2}{*}{ Bos taurus } & Bt nxf1 & 29 & $10-11$ & + & 1810 \\
\hline & Bt $n x f 3$ & $\mathrm{X}$ & $9-10$ & - & 569 \\
\hline Sus scrofa & Ss $n x f 1$ & 2 & $10-11$ & $?$ & 1799 \\
\hline Oryctolagus cuniculus & Oc $n x f 1$ & NW_003159343 & $10-11$ & $?$ & 1811 \\
\hline Ailuropoda melanoleuca & $A m n x f 1$ & NW_003218217 & $10-11$ & $?$ & 1823 \\
\hline Callithrix jacchus & Cj $n x f 1$ & 11 & $10-11$ & $?$ & 1828 \\
\hline \multirow[t]{3}{*}{ Nomascus leucogenys } & $N l n x f$ & scaffold_99 & $10-11$ & $?$ & 1804 \\
\hline & Hs nxf1 & 11 & $10-11$ & $+{ }^{* * *}$ & 1801 \\
\hline & $H s n x f 2$ & $\mathrm{X}$ & $12-13$ & - & 1678 \\
\hline \multirow[t]{3}{*}{ Homo sapiens } & Hs nxf3 & $\mathrm{X}$ & $9-10$ & + & 1642 \\
\hline & Hs nxf4 & $\mathrm{X}$ & $9-10$ & - & 1682 \\
\hline & Hs $n x f 5$ & $\mathrm{X}$ & $10-11$ & - & 1672 \\
\hline
\end{tabular}

Information sources: "Ivankova et al. 2010; ${ }^{* *}$ Sasaki et al. 2005; ${ }^{* * *}$ Li et al. 2006; FlyBase; Genbank; UCSC Genome.

[14]. Later, TAP was demonstrated to be involved in the nuclear-cytoplasmic transport of the unspliced or partially spliced RNA of retroviruses. TAP directly recognizes only one sequence-CTE (Constitutive Transport Element)-which was initially discovered in RNAs of retroviruses [15-18]. Adaptor proteins mediate the interaction between NXF1 and cellular mRNAs in metazoan [19-21]. Nuclear mRNA export is connected with transcription, splicing, processing, and mRNA quality control [12].

\subsection{Modular Principle of Organization of NXF Factors}

Proteins of the NXF family have a modular domain or- ganization consisting of an RNA-binding domain (RBD), four leucine-rich repeats (LRRs), a domain exhibiting a similarity to the nuclear transport factor 2 (NTF2-like domain), and a C-terminal ubiquitin associated (UBA)-like domain (Figure 2) [22,23]. These proteins are considered RNA transport receptors due to the combination of their receptor and transport functions. The N-terminus of the protein is predominantly responsible for interacting with mRNA, while the C-terminus enables transport of the RNP complex through nuclear pores by interacting with partner protein p15 and nucleoporins - proteins of nuclear pore complexes [24,25]. The RBD (RNA-binding domain) belongs to the RRM (RNA recognition motif) family, which has a distinctive $\beta \alpha \beta \beta \alpha \beta$ structure, 


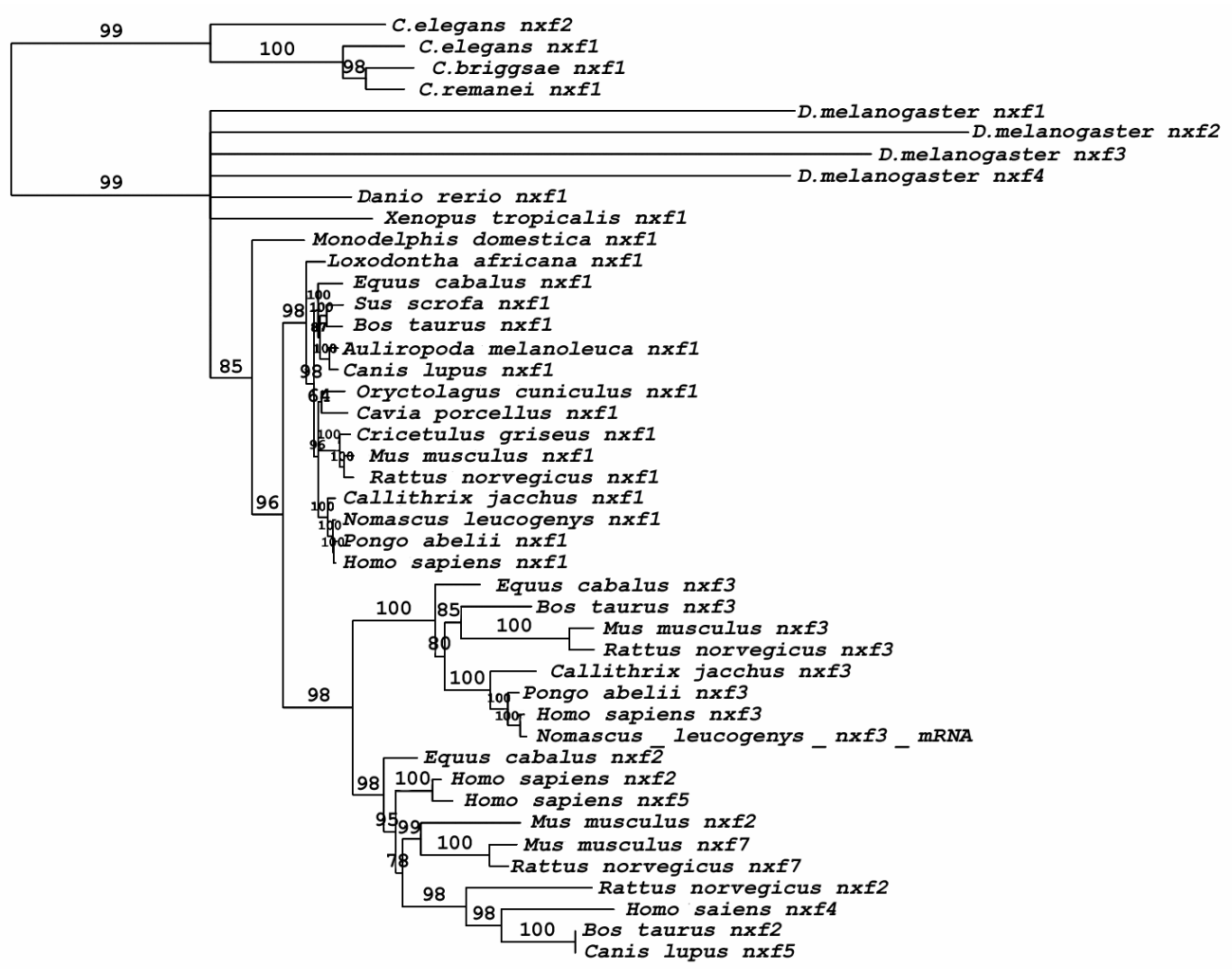

Figure 1. Phylogenetic tree of NXF family sequences, constructed for species of the phyla Nematoda, Arthropoda, and Chordata. The tree was constructed for sequences of ubiquitous transcripts. The tree was drawn by the MrBayes program (2,000,000 generations). Numbers denote posterior clade probabilities.

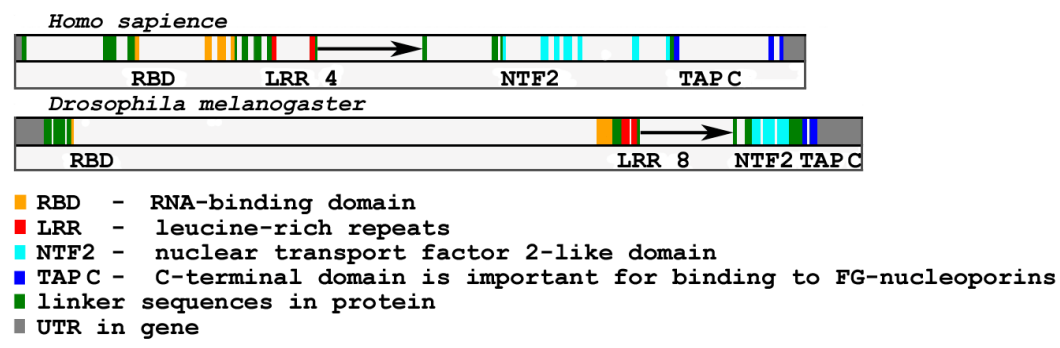

Figure 2. Intron-exon structure of $H s n x f 1$ and $D m n x f 1$ genes. Arrow shows cassette intron. The color of exons corresponds to color of domains in protein.

characteristic of many RNA-binding proteins, such as $\mathrm{PAB}$ - poly(A)-binding-protein, Sex-lethal protein (Sxl), and U1A and U2B" splicing factors [16]. The receptor function is much older: RRM have been discovered in proteins of prokaryotes [26]. While the RRM domain alone is sufficient for binding with mRNA in vitro, both RRM and LRR are necessary for exporting mRNA in vivo $[16,23]$.

The C-terminal end of NXF proteins is represented by NTF2-like and UBA-like domains. The NTF2-like domain is thought to be derived from a prokaryotic precursor [27]. The C-terminal end in proteins paralogous to NXF often differs from the corresponding parts in NXF1 proteins. Consequently, many paralogous proteins, such as Dm NXF3, Dm NXF4, Ce NXF2, Hs NXF3, Hs NXF5, Mm NXF3, and Mm NXF7 are incapable of binding with nucleoporins $[1,8,11,28-30]$. In most cases this is due to the loss of a UBA-like domain in paralogous proteins, or the absence of both domains (as in the case of the Dm NXF4 protein) [1,7,31].

The unification of the transport and receptor functions probably took place simultaneously with the origin of the eukaryotic cell. In eukaryotes, transcription and mRNA processing are physically and temporally separated from translation by the nuclear membrane, which required the formation of mechanisms for the active transport of macromolecules, including mRNAs, from the nucleus to the cytoplasm. Most transcripts begin translation soon 
after they exit the nucleus. The specific class of transcripts capable of staying for extended periods of time in the cytoplasm in a state unavailable for translation presumably required specialization of transport receptors. There are paralogous genes that, unlike the universal gene $n x f 1$, usually exhibit an organ-specific character of expression in mammals $[1,8]$. The products of paralogous genes are usually localized in the cytoplasm $[8,11$, $28,30,32]$.

Several processes of transformation of genetic material underlie the formation of gene families: duplication, separation of certain genes, or fusion of different parts of genes [33-36]. The existence of clusters of linked genes in different organisms seems to support the hypothesis that the $n x f$ gene family was formed via duplication $[1,8]$.

Integration of cDNA copies of transcripts into a genome may also produce gene copies that contain no introns [36-38]. Perhaps this mechanism eventually produced genes $D m n x f 3$ and $D m n x f 4$, which contain no introns in D. melanogaster. It is important to note, however, that the lack of introns in genes that are orthologous to $D m n x f 4$ is not characteristic of all species of the genus Drosophila for which it has been described (FlyBase, 2012).

LLR and NTF2-like domains responsible for the interaction of NXFs with various partner proteins are the most highly conserved [39].

\subsection{Interaction of NXF1 Proteins with RNA}

Nuclear mRNAs are exported as RNP-complexes [40]. NXF1 interacts with mRNA by means of adaptor proteins, which are part of mRNP complexes during transcription, processing, and transport [25,41-43]. During mRNA export, NXF1 partners with proteins E1B-AP5, RAE1, and members of evolutionarily conserved families of proteins-Yralp in yeast and REF in mammals $[24,44,45]$, as well as components of the TREX (TRanscription-EXport) complex [40,43].

Most genes of eukaryotes have introns that are then deleted from pre-mRNA during splicing. As a rule, cellular mRNAs with introns do not leave the nucleus $[16,46]$. Translation of incompletely spliced mRNA due to the presence of a premature termination codon may produce truncated proteins, which are potentially deleterious to a cell. In the nucleus, transcripts undergo quality control, and normally only completely spliced mRNAs are capable of export $[47,48]$.

Genes that correspond to transcripts with intron retention are not uncommon in humans [49]. The expression of intron-containing messages has been shown to occur in a variety of diseases including several cancers [50], and also as a response to vascular injury in rats [51]. Transcripts with retained introns may serve as sources of alternative protein products with an independent function that may, among other things, influence the function of a full-length product [52].

If mRNAs with retained introns are abundant in the cytoplasm, intron retention is probably regulated by factors involved in both splicing and mRNA export. Some of the retroviruses have been shown to export unspliced RNA by means of cis-acting RNA elements, termed constitutive transport elements (CTEs), which interact directly with cellular export proteins [53]. Export of mRNA with retained introns in simple retroviruses is carried out with the help of NXF1 (TAP), which binds directly to the CTE sequence in the genome of retroviruses [15-18,24,53,54]. Microinjection experiments performed on Xenopus oocytes have demonstrated that TAP (Hs NXF1) directly interacts with the CTE, allowing the export of CTE-containing RNAs [15]. Moreover, TAP remains bound to CTE-containing RNAs in polyribosomes and may be present inside the nucleus or in the nuclear rim, as well as in the cytoplasm [55].

\section{RESULTS AND DISCUSSIONS}

\subsection{Alternative Intron-Retaining Transcripts of nxf1 Genes}

Both the domain structure and the intron-exon structure of the NXF family proteins are evolutionarily conserved. Among the known $n x f$ genes, most have an intron-exon structure. Mex67 in fungi, nxf4 in some species of the genus Drosophila, and Dm nxf3 in D. melanogaster, have no introns.

A feature specific to $n x f l$ genes is the existence of alternative transcripts with a retained intron flanked by evolutionarily conserved exons: $110 \mathrm{nt}$ upstream and 37 nt downstream of the respective intron. This intron from the evolutionarily conserved block, which can be excised or retained in alternative transcripts of $n x f 1$, we named a "cassette" intron. Such an evolutionarily conserved exonintron block is characteristic of most of the $n x f$ family genes that exhibit an intron-exon structure. There exist $n x f$ genes in which the sequences of exons $110 \mathrm{nt}$ and 37 nt are not separated by an intron, and are represented by exon 147 nt: $D m n x f 2$ and $C e n x f 2$, for example. Transcripts of $n x f 1$ genes with a cassette intron between exons $110 \mathrm{nt}$ and $37 \mathrm{nt}$ have been shown for $n x f 1$ genes in M. musculus [8], H. sapiens [56], and D. melanogaster [57]. Such $n x f 1$ transcripts have been found in other species, as well. ESTs (expressed sequence tags) include a portion of one of the aforementioned exons and a portion of the cassette intron (Genbank, UCSC) (see Table 1).

We have demonstrated that the transcript with cassette intron 5 - 6 in the head tissues of adult fruit flies exceeds the universal, completely spliced transcript of the gene Dm $n x f 1$, in its relative content (Figure 3) [57]. Usually, 
intron-containing transcripts stay in the nucleus $[46,48]$. Even if they enter the cytoplasm, they are subject to the cytoplasmic mRNA quality control mechanism (surveillance) termed NMD (nonsense-mediated mRNA decay) [58,59]. The large number of transcripts of $n x f 1$ genes with a retained intron in different organisms raises the following questions:

1) How do intron-containing transcripts manage to exit the nucleus, bypassing the mRNA quality control mechanism?

2) What makes the intron-containing transcripts immune to NMD in the cytoplasm?

3) What evolutionarily conserved features characterize cassette introns in alternative intron-containing transcripts of $n x f 1$ genes?

Analyzing specific features of the intron retained in alternative transcripts of $n x f 1$ genes may help answer these questions. The existence of a sequence with a length of about $100 \mathrm{nt}$, which resembles the CTE of the MPMV virus in cassette introns 10 - 11, represented in genes $H s \quad n x f 1$ and $M m n x f 1$, has been observed [56]. This sequence is highly conserved in cassette introns of $n x f 1$ in various animals (Figure 4). $H s n x f 1$ mRNA with the retained intron is exported from the nucleus and is represented in a polysomic fraction [56]. Due to the presence of a premature stop-codon at the beginning of the cassette intron 10 - 11, translation results in a truncated protein. A similar truncated protein has been discovered in human cells [56]. The CTE sequence is an important element affecting the expression of intron-retaining mRNAs in mammals $[60,61]$. The CTE has not been discovered in the corresponding introns of paralogous genes of the $n x f$ family in mice and humans.

When comparing introns 5 - 6 of $n x f l$ genes in different

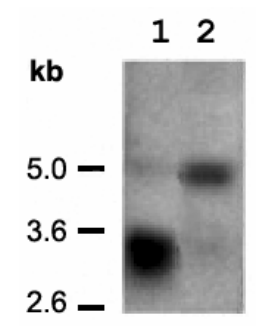

Figure 3. Northern blot analysis of total RNA from different tissues of the adult Drosophila female. 1-ovaries, 2 - heads. The sizes (in $\mathrm{kb}$ ) of molecular weight RNA markers are indicated on the left (Ivankova et al., 2010). species of Drosophila, we have not discovered extended homologous sequences (such as cassette introns in genes orthologous to the $n x f 1$ of vertebrates). However, we have found a common feature of introns 5 - 6 of the $n x f 1$ gene in different species of Drosophila: the presence of two poly(A) sequences, each with a length of around 100 nt (Figure 5). This feature accounts for the difference of cassette introns 5 - 6 of $n x f 1$ genes in Drosophilidae from cassette introns 10 - 11 of $n x f 1$ genes in vertebrates.

The cassette introns of $n x f 1$ in Drosophilidae form complex secondary structures. Poly(A) sequences of introns 5 - 6 of $n x f 1$ in Drosophilidae is usually located in loops (Figure 6). Because the same RNA sequence can form several secondary structure variants, Figure 6 depicts only the most probable structures of introns $5-6$ of $n x f 1$ genes in some Drosophila species. The choice of species was made in accordance with the relationships between Drosophila species, taking into consideration the phylogenetic tree constructed with the divergence of sequences of intron 5 - 6 (Figure 7).

What happens to the poly(A) RNA sequence? The presence of a poly(A) tail is an important element in RNA export [62]. Export efficiency depends on the length of this sequence. The poly(A) sequence is responsible for binding with corresponding proteins [63]. The poly(A) sequence is believed to be a recognition target for export factors [62].

Taking into account the secondary structure of cassette introns of $n x f 1$ genes in Drosophilidae, poly(A) sequences are probably open for interaction with proteins that may recognize them. Poly(A) sequences may not only serve as nuclear export markers, but may also protect the corresponding transcript from degradation in the cytoplasm. It has been demonstrated that the protein PABPC1 (cytoplasmic poly(A)-binding protein) suppresses NMD in D. melanogaster [64].

\subsection{Specific Features of Retained Introns in Alternative Transcripts of $n x f$ Genes}

Cassette introns of $n x f 1$ genes in different species of mammals form complex secondary structures, as well as cassette introns in Drosophilidae $n x f 1$ (Figure 8). It is possible that this feature of cassette introns determines the fate of intron-retaining transcripts in the cytoplasm. It is also possible that cassette introns of $n x f l$ genes have an independent function both inside the intron-retained transcript, and as the product of splicing of the premRNA of the $n x f 1$. RNAs transcribed from introns are known to participate in a number of processes related to post-transcriptional control of gene expression [65].

Cassette introns of $n x f l$ genes in vertebrates have four evolutionarily conserved regions (Figure 4). The first, with a length of around $130 \mathrm{nt}$, is the 5'-end of cassette introns (Figure 4). This sequence includes 17 complete 
A

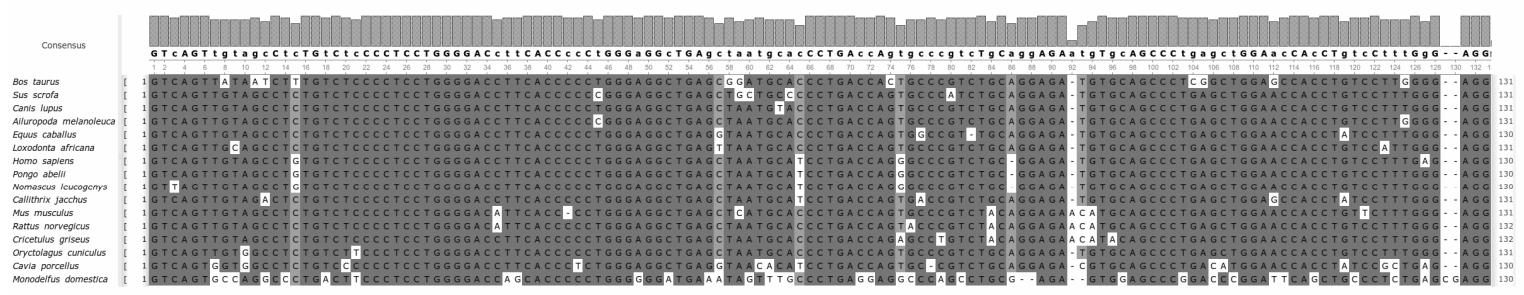

в
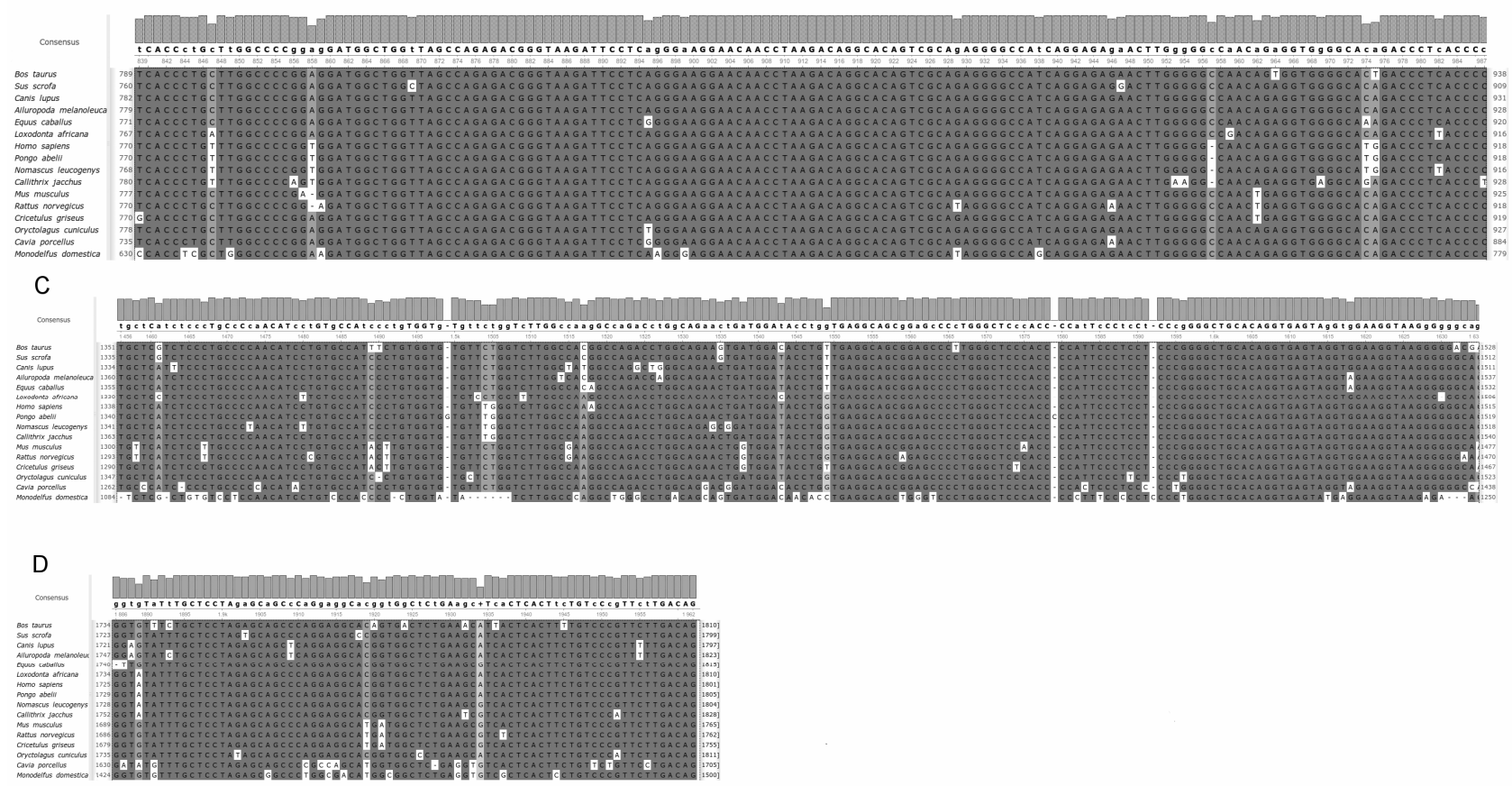

Figure 4. Long evolutionarily conserved regions of $n x f 1$ cassette introns: (A) 5 '-end of introns; (B) CTE sequences; (C) third conserved sequences; (D) 3'-ends of introns. Species, top to bottom: Bos taurus, Sus scrofa, Canis lupus familiaris, Auliropoda melanoelica, Equus caballus, Loxodonta africana, Homo sapiens, Pongo abelii, Nomascus leucogenys, Callithrix jacchus, Mus musculus, Rattus norvegicus, Cricetulus grizeus, Oryctolagus cuniculus, Cavia porcellus, Monodelphis domestica.

$\boldsymbol{A}$

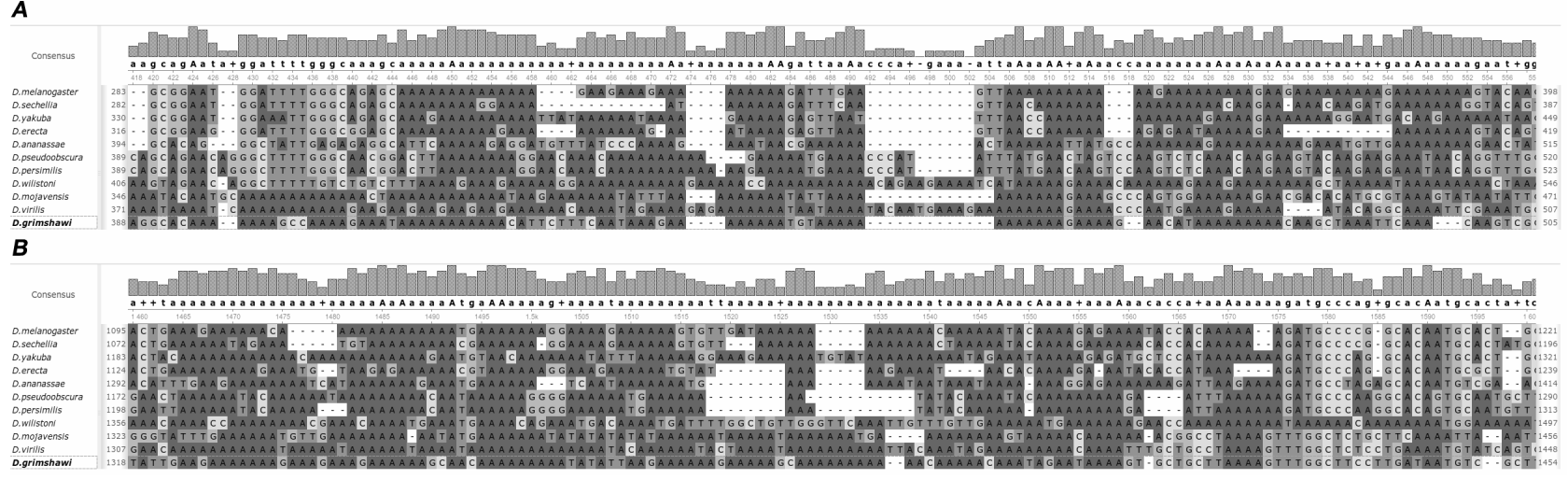

Figure 5. Poly(A) sequences in cassette introns 5 - 6 of the $n x f 1$ genes in different species of Drosophila. (A) First and (B) second poly(A) sequences. Species, top to bottom: D. melanogaster, D. sechellia, D. yakuba, D. erecta, D. ananassae, D. pseudoobscura, D. persimilis, D. wilistoni, D. mojavensis, D. virilis, D. grimshawi.

codons that continue the open reading frame of the preceding exon 10 . The last 17 amino acids in the short pro- tein that corresponds to the $H s$ nxfl transcript with retained intron $10-11[56]$ is evolutionarily conserved in 


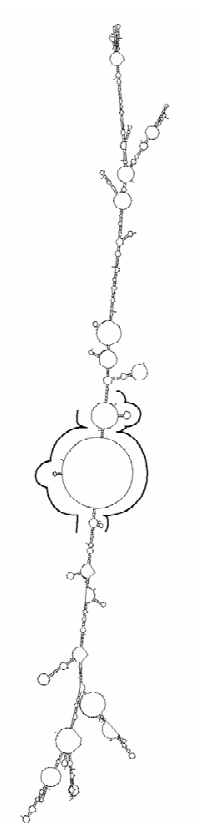

D.ananassae

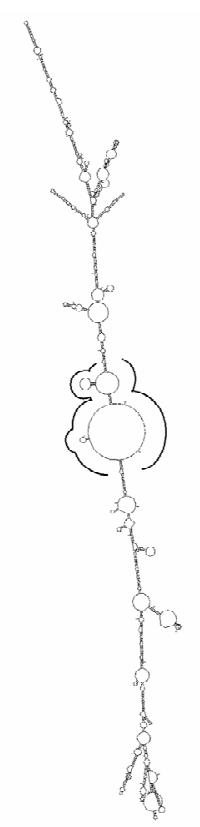

D.grimshawi

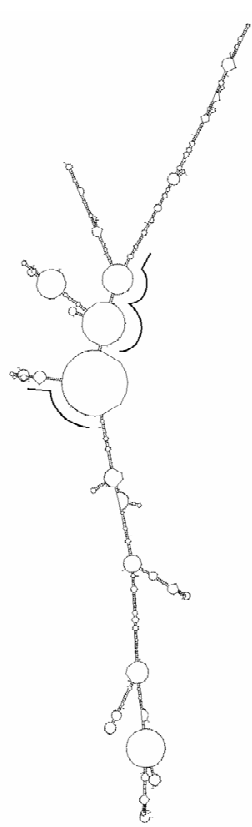

D. melanogaster

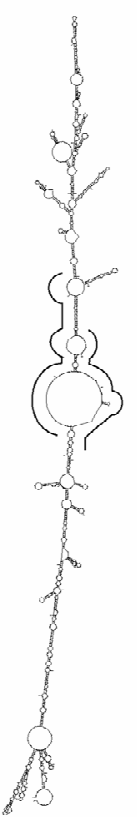

D. pseudoobscura

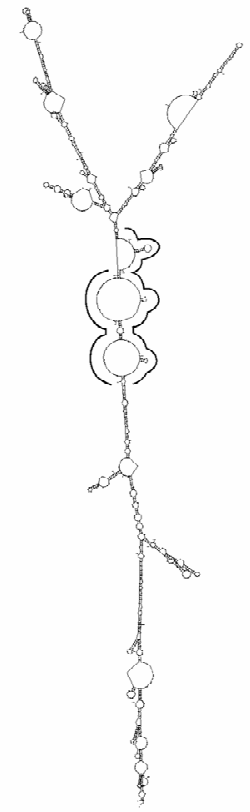

D. yakuba

Figure 6. Secondary structures of cassette introns $5-6$ of the $n x f 1$ genes in five species of Drosophila. Marked loops, formed using poly(A) sequences.

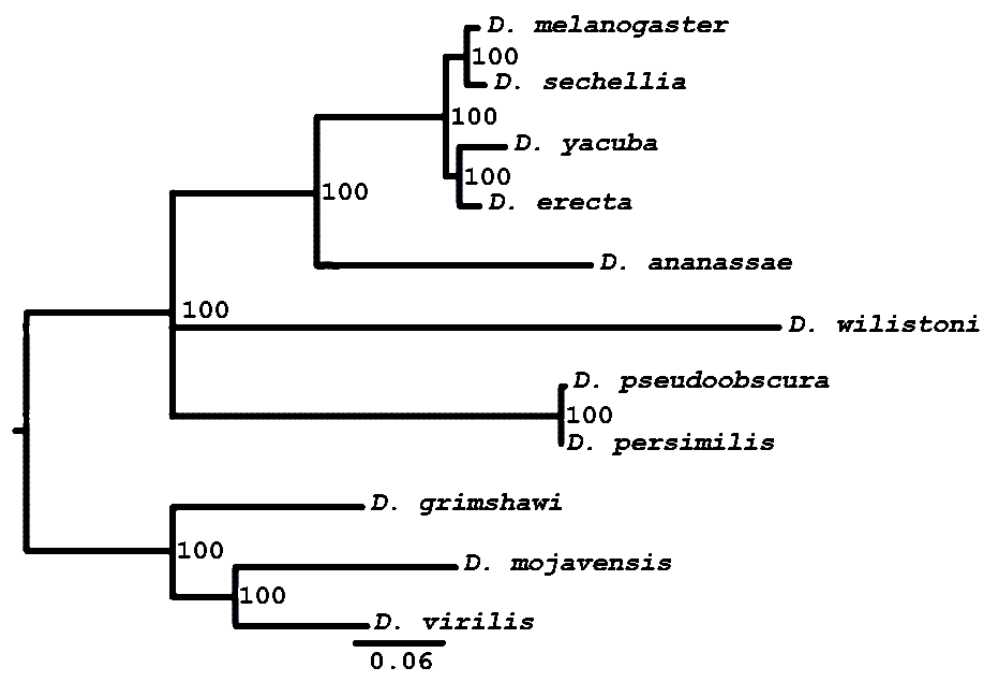

Figure 7. Phylogenetic tree constructed using divergences of the sequences of cassette intron 5 - 6 of $n x f 1$ genes in eleven species of Drosophila.

truncated proteins corresponding to the intron-containing transcripts in other vertebrates (Figure 9). The first stop-codon in intron $10-11$ is duplicated by the next one, $12 \mathrm{nt}$ down. Evolutionary conservation of the first region (Figure 4(a)) suggests the functional significance of the truncated NXF1. The only species that is not present in the list is the M. musculus. Deleting one of the five nucleotides (C) at the position 40 - 44 of $M m n x f l$ intron shifts the open reading frame compared to other species, and the C-terminus of the supposed truncated protein corresponds to the 80th full codon in intron $10-11$ of Mm nxf1.

The first conserved region of cassette introns (Figure 4(a)) is partially complimentary to the fourth conserved region (Figure 4(d)), which is at the 3'-end of the intron. In secondary RNA structures the first and fourth regions form a "stem", thus "closing" the secondary structure of cassette introns 10 - 11 (Figure 8). 
The second evolutionarily conserved sequence (Figure 4(b)) contains CTE and has been identified by $\mathrm{Li}$ with co-authors [56]. Whereas this sequence does not contain an ORF (open reading frame), the third evolutionarily conserved sequence (Figure 4(c)) in introns 10 - 11 of the genes $H s n x f 1$ and $M m n x f 1$ is a long open reading frame.

The supposed proteins, which correspond to the open reading frame in the third evolutionarily conserved sequence of introns 10 - 11 of the genes $H_{s} n x f l$ and $M m$ $n x f 1$, exhibit a great degree of similarity (data not provided). If the open reading frame is significant, it may be preserved in other cassette introns of $n x f 1$ genes in vertebrates. This, however, we did not observe when evaluating the third evolutionarily conserved region of $n x f 1$ genes in other vertebrates for the presence of a long evolutionarily conserved ORF. In all the depicted secondary structures of cassette introns, region three is a hairpin (Figure 8), which supports the assumption of a structural role of this evolutionarily conserved region.

Long non-coding RNAs often contain ORF. It is speculated that such sequences can be translated, but with very low efficiency, or only at a specific stage of development [66]. Little is known about ncRNAs that code for functionally significant short proteins [67]. Potentially, the possibility for synthesizing short peptides also exists for cassette introns of $n x f 1$ genes. Short peptides, according to some researchers [68], may be a new class of bioactive signaling molecules.

Many transcripts, including those that code for a protein, also carry out regulatory functions. They are capable of interactions via a specific nucleotide sequence, thus playing a structural role or serving as catalysts $[66,69]$. Evolutionarily conserved sequences in the cassette intron of $n x f 1$ genes raise the questions of what adaptive advantages these sequences bring, and what the functional significance of intron-retained transcripts is. The evolutionary path of forming adaptive features may vary depending on the characteristics of a particular taxon.

The existence of sequences that may facilitate export of transcripts containing these introns from the nucleus to the cytoplasm is a feature shared between introns 10 11 in $n x f 1$ genes in vertebrates and introns $5-6$ in $n x f 1$ genes of different species of Drosophila. This, along with the ability of these introns to form secondary structures,
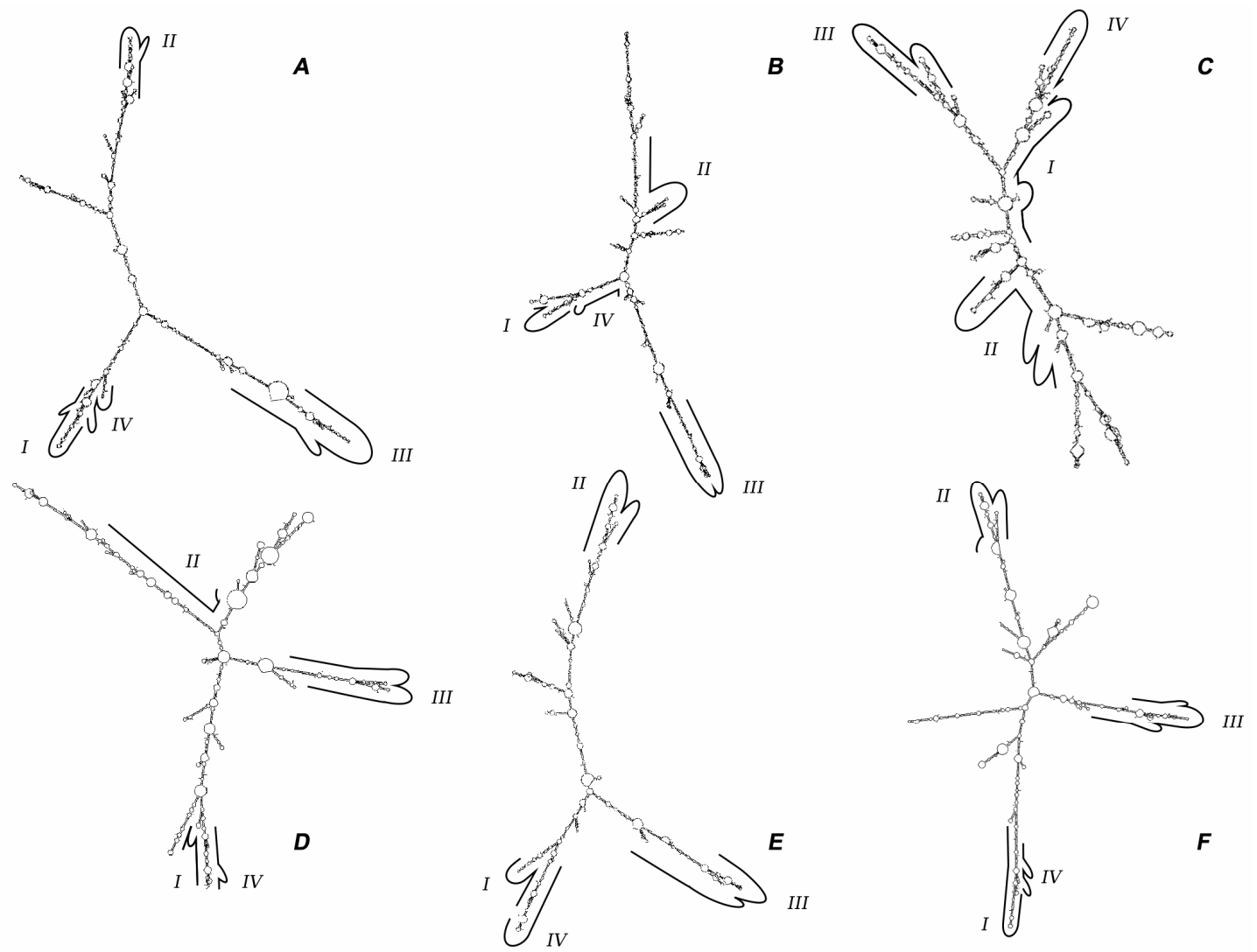

Figure 8. Secondary structures corresponding to the minimum energy state of the cassette intron of the $n x f 1$ gene in different species of mammals. A-Callithrix jacchus; B-Canis lupus familiaris; C-Monodelphis domestica; DLoxodontha africana; E-Rattus norvegicus; F-Equus cabalus. Roman numerals denote evolutionarily conserved regions: I—5'-end of intron, II—CTE sequence, III — third conserved sequence, IV—3'-end of intron. 


\begin{tabular}{|c|c|c|c|c|c|c|c|c|c|c|c|c|c|c|c|c|c|c|c|}
\hline \multirow[t]{2}{*}{ Consensus } & \multicolumn{19}{|c|}{ SVVasvsPPGD I HPIGG $x$ a nap $x$} \\
\hline & & 12 & & 4 & 6 & & 8 & & 10 & & 12 & 14 & & 16 & 18 & & 20 & & 23 \\
\hline Bos_taurus & {$[1]$} & S V & I & I $\mathrm{F}$ & & 15 & $\mathbf{P}$ & $P$ & G & D I & L & 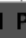 & I & $\mathbf{G}$ & $\mathrm{G} X$ & & D & A P & $\mathbf{P} \times$ \\
\hline Sus_scrofa & {$[1$} & S V & V & A 5 & $5 \mathrm{~V}$ & 5 & $\mathbf{P}$ & $\mathbf{P}$ & & D 1 & L & & $\mathrm{P}$ & G & $G x$ & & & A P & $\mathbf{P X}$ \\
\hline Canis_lupus & {$\left[\begin{array}{l}1 \\
{[}\end{array}\right.$} & s v & $v$ & A 5 & $5 \mathrm{~V}$ & 5 & P & $P$ & & D 1 & L & & $\mathrm{L}$ & G & $G \times$ & & & $\mathrm{VP}$ & $\mathbf{P} \times$ \\
\hline Alluropoda_melanoleuca & {$\left[\begin{array}{l}1 \\
0\end{array}\right.$} & S V & V & A 5 & $5 \mathrm{~V}$ & 5 & P & $\mathbf{P}$ & G & D 1 & L $\mathbf{r}$ & & $\mathrm{P}$ & G & $G \times$ & A & & A P & $\mathbf{P} \times$ \\
\hline Equus_caballus & {$\left[\begin{array}{l}1 \\
{[}\end{array}\right.$} & S V & $\mathrm{V}$ & A 5 & & 5 & P & $\mathbf{P}$ & G & D 1 & L & & $\mathbf{L}$ & G & $G \times$ & & & A P & $P X$ \\
\hline Loxodonta_africana & {$\left[\begin{array}{l}1 \\
{[}\end{array}\right.$} & s v & A & A 5 & & 5 & $P$ & $\mathbf{P}$ & G & D 1 & L & & $\mathbf{L}$ & & $G \times$ & V & & A P & $P \times$ \\
\hline Homo_sapiens & {$\left[\begin{array}{l}1 \\
{[}\end{array}\right.$} & s v & V & $A C$ & & 5 & $\mathrm{P}$ & $\mathbf{P}$ & G & D 1 & L r & & $\mathbf{L}$ & G & $G \times$ & A & & A S & \\
\hline Pongo_abelli & {$\left[\begin{array}{l}1\end{array}\right]$} & S V & V & $A C$ & & 15 & $\mathbf{P}$ & P & & D 1 & L & & L & & $G \times$ & & & A S & \\
\hline Nomascus_leucogenys & {$[1]$} & L V & V & $A C$ & & 5 & P & $\mathbf{P}$ & & D & L & & L & & $G \times$ & & & A S & $S x$ \\
\hline Callithrix_jacchus & {$\left[\begin{array}{l}1 \\
{[}\end{array}\right.$} & s v & v & D 5 & & 5 & $\mathbf{P}$ & $\mathbf{P}$ & G & D & L & & $L$ & & $G \times$ & A & & & $S x$ \\
\hline Rattus_norvegicus & {$\left[\begin{array}{l}1 \\
{[}\end{array}\right.$} & S V & V & A 5 & & 5 & F & $\mathbf{P}$ & G & D & I & & L & & $G \times$ & A & & A P & $P X$ \\
\hline Cricetulus_griseus & [ 1 & s v & v & A 5 & & 5 & P & $\mathbf{P}$ & & D & L & & L & G & $G X$ & & & A P & $P X$ \\
\hline Oryctolagus_cuniculus & [ 1 & s v & v & A S & & $F$ & & $\mathbf{P}$ & & D & L & & & & & & & & $P X$ \\
\hline Cavia_porcellus & {$\left[\begin{array}{l}1 \\
0\end{array}\right.$} & s V & 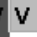 & A 5 & & $P$ & & $\mathbf{P}$ & & D 1 & & & & & $G \times$ & & & $\mathrm{T} S$ & $s x$ \\
\hline Monodelfus_domestica & {$\left[\begin{array}{l}1 \\
{[}\end{array}\right]$} & S V & & & & & & P & G & & Q & I F & & & $\mathrm{G} X$ & & & $\mathbf{L} \mathbf{P}$ & $\mathbf{P}:$ \\
\hline
\end{tabular}

Figure 9. C-terminal end of the short isoform of the NXF1 protein in mammals, which is presumably translated from an alternative transcript containing unspliced intron $10-11$.

suggests the functional significance of such transcripts. It is possible that the retention of the cassette intron in transcripts of $n x f l$ genes facilitates the synthesis of the truncated protein NXF1. We have shown that in the heads of adult flies the transcript with cassette intron 5 6 is more abundant than completely spliced transcripts (Figure 3) [57]. It would be interesting to test the hypothesis about the existence of the truncated protein Dm NXF1 specific to the brain tissues of the fruit fly.

\section{CONCLUSION}

A great variety of alternative transcripts, including intron-retained and non-coding RNAs, is characteristic of the nervous system. Thus, it is not surprising that transcripts with cassette intron 5 - 6 in $D m n x f 1$ are found primarily in the head of fruit flies. Insects that are characterized by complex behavior are model organisms, which facilitate the study of the molecular mechanisms of the nervous system. The large number of transcripts with retained intron 5 - 6 of the $D m n x f 1$ gene in the head of adult fruit flies points to the functional significance of these transcripts. The existence of evolutionarily conserved sequences in the cassette introns of $n x f l$ genes in animals within some taxonomic groups can be regarded as the acquisition of adaptive properties of the corresponding intron-containing transcripts. These sequences may provide benefits in nuclear-cytoplasmic transport, resistance to NMD, and a possible involvement in the regulation of gene expression. The ability of cassette introns to form complex secondary structures suggests that these introns may have an independent, possibly structural, function and also be a source of non-coding RNAs.

\section{METHODS}

For the analysis of nucleotide sequences we used the Unipro UGENE v1.10.0 software suite

(http://ugene.unipro.ru) [70] along with third-party tools: ClustalW (http://www.clustal.org/clustal2/) [71] and TCoffee (http://www.tcoffee.org/Projects/tcoffee/) [72] alignment algorithms.

The tree structure was built using the MrBayes program $(2,000,000$ generations $)$

(http://mrbayes.sourceforge.net/index.php) [73]. To view the phylogram in a more convenient form we used the FigTree v1.3.1 editor (http://tree.bio.ed.ac.uk/software/figtree/).

To build the secondary structures of nucleotide sequences we used the UNAFold v3.8 suite

(http://mfold.rna.albany.edu/). Secondary structure prediction was based on the minimum free energy calculation [74].

The following databases were used:

Genbank (http://www.ncbi.nlm.nih.gov/)

Flybase (http://flybase.org/)

UCSC Genome (http://genome.ucsc.edu)

\section{ACKNOWLEDGEMENTS}

Our work is supported by the Leading Scientific Schools program (SciSch-6455.2010.4; SciSch-5345.2012.4), the Russian Foundation for Basic Research (projects 09-04-00697 and 12-04-00934), Federal Target Program (02.740.11.0698).

\section{REFERENCES}

[1] Herold, A., Suyama, M., Rodrigues, J.P., Braun, I.C., 
Kutay, U., Carmo-Fonseca, M., Bork, P. and Izaurralde, E. (2000) TAP (NXF1) Belongs to a multigene family of putative RNA export factors with a conserved modular architecture. Molecular and Cellular Biology, 20, 89969008. doi:10.1128/MCB.20.23.8996-9008.2000

[2] Wilkie, G.S., Zimyanin, V., Kirby, R., Korey, C., Francis-Lang, H., Van Vactor, D. and Davis, I. (2001) Small bristles, the Drosophila ortholog of NXF-1, is essential for mRNA export throughout development. RNA, 7, 1781-1792.

[3] Herold, A., Teixeira, L. and Izaurralde, E. (2003) Genome-wide analysis of nuclear mRNA export pathways in Drosophila. EMBO Journal, 22, 2472-2483. doi:10.1093/emboj/cdg233

[4] Hurt, E., Strässer, K., Segref, A., Bailer, S., Schlaich, N., Presutti, C., Tollervey, D. and Jansen, R. (2000) Mex67p mediates nuclear export of a variety of RNA polymerase II transcripts. The Journal of Biological Chemistry, 275, 8361-8368. doi:10.1074/jbc.275.12.8361

[5] Serpeloni, M., Vidal, N., Goldenberg, S., Avila, A.R. and Hoffmann, F.G. (2011) Comparative genomics of proteins involved in RNA nucleoplasmic export. BMC Evolutionary Biology, 11, 7.

http://www.biomedcentral.com/1471-2148/11/7 doi:10.1186/1471-2148-11-7

[6] Segref, A., Sharma, K., Doye, V., Hellwig, A., Huber, J., Lührmann, R. and Hurt, E. (1997) Mex67p, a novel factor for nuclear mRNA export, binds to both poly(A)+ RNA and nuclear pores. EMBO Journal, 16, 3256-3271. doi:10.1093/emboj/16.11.3256

[7] Herold, A., Klymenko, T. and Izaurralde, E. (2001) NXF1/p15 heterodimers are essential for mRNA nuclear export in Drosophila. RNA, 7, 1768-1780.

[8] Sasaki, M., Takeda, E., Takano, K., Yomogida, K., Katahira, J. and Yoneda, Y. (2005) Molecular cloning and functional characterization of mouse $N x f$ family gene products. Genomics, 85, 641-653. doi:10.1016/j.ygeno.2005.01.003

[9] Tan, W., Zolotukhin, A.S., Bear, J., Patenaude, D.J. and Felber, B.K. (2000) The mRNA export in Caenorhabditis elegans is mediated by Ce-NXF-1, an ortholog of human TAP/NXF and Saccharomyces cerevisiae Mex67p. RNA, 6, 1762-1772. doi:10.1017/S1355838200000832

[10] Tan, W., Zolotukhin, A.S., Tretyakova, I., Bear, J., Lindtner, S., Smulevitch, S.V. and Felber, B.K. (2005) Identification and characterization of the mouse nuclear export factor $(N x f)$ family members. Nucleic Acids Research, 33, 3855-3865. doi:10.1093/nar/gki706

[11] Jun, L., Frints, S., Duhamel, H., Herold, A., Abad-Rodrigues, J., Dotti, C., Izaurralde, E., Marynen, P. and Froyen, G. (2001) NXF5, a novel member of the nuclear RNA export factor family, is lost in a male patient with a syndromic form of mental retardation. Current Biology, 11, 1381-1391. doi:10.1016/S0960-9822(01)00419-5

[12] Köhler, A. and Hurt, E. (2007) Exporting RNA from the nucleus to the cytoplasm. Nature Reviews Molecular Cell Biology, 8, 761-773. doi:10.1038/nrm2255

[13] Yao, W., Roser, D., Köhler, A., Bradatsch, B., Baßler, J. and Hurt, E. (2007) Nuclear export of ribosomal 60S subunits by the general mRNA export receptor Mex67Mtr2. Molecular Cell, 26, 51-62. doi:10.1016/i.molcel.2007.02.018

[14] Yoon, D.W., Lee, H., Seol, W., DeMaria, M., Rosenzweig, M. and Jung, J.U. (1997) Tap: A novel protein that interacts with tip of herpesvirus saimiri and induces lymphocyte aggregation. Immunity, 6, 571-582. doi:10.1016/S1074-7613(00)80345-3

[15] Grüter, P., Tabemero, C., von Kobbe, C., Schmitt, C., Saavedra, C., Bachi, A., Wilm, M., Felber, B.K. and Izaurralde, E. (1998) TAP, the human homolog of Mex67p, mediates CTE-dependent RNA export from the nucleus. Molecular Cell, 1, 649-659. doi:10.1016/S1097-2765(00)80065-9

[16] Liker, E., Fernandez, E., Izaurralde, E. and Conti, E. (2000) The structure of the mRNA nuclear export factor TAP reveals a cis arrangement of a non-canonical RNP domain and an LRR domain. EMBO Journal, 19, 55875598. doi:10.1093/emboj/19.21.5587

[17] Zolotukhin, A.S., Michalowski, D., Smulevitch, S. and Felber, B.K. (2001) Retroviral constitutive transport element evolved from cellular TAP(NXF1)-binding sequences. Journal of Virology, 75, 5567-5575. doi:10.1128/JVI.75.12.5567-5575.2001

[18] Teplova, M., Wohlbold, L., Khin, N.W., Izaurralde, E. and Patel, D.J. (2012) Structure-function studies of nucleocytoplasmic transport of retroviral genomic RNA by mRNA export factor TAP. Nature Structural and Molecular Biology, 18, 990-998. doi:10.1038/nsmb.2094

[19] Gatfield, D. and Izaurralde, E. (2002) REF1/Aly and the additional exon junction complex proteins and dispensable for nuclear mRNA export. The Journal of Cell Biology, 159, 579-588. doi:10.1083/jcb.200207128

[20] Huang, Y., Gattoni, R., Stevenin, J. and Steitz, J.A. (2003) SR splicing factors serve as adapter proteins for TAPdependent mRNA export. Molecular Cell, 11, 837-843. doi:10.1016/S1097-2765(03)00089-3

[21] Hautbergue, G.M., Hung, M.-L., Golovanov, A.P., Lian, L.-Y. and Wilson, S.A. (2008) Mutually exclusive interaction drive handover of mRNA from export adaptor to TAP. Proceedings of the National Academy of Sciences of the USA, 105, 5154-5159. doi:10.1073/pnas.0709167105

[22] Bear, J., Tan, W., Zolotukhin, A.S., Tabernero, C., Hudson, E.A. and Felber, B.K. (1999) Identification of novel import and export signals of human TAP, the protein that binds to the constitutive transport element to the type D retrovirus mRNAs. Molecular and Cellular Biology, 19, 6306-6317.

[23] Katahira, J., Strässer, K., Podtelejnikov, A., Mann, M., Jung, J.U. and Hurt, E. (1999) The Mex67p-mediated nuclear mRNA export pathway is conserved from yeast to human. EMBO Journal, 18, 2593-2609. doi:10.1093/emboj/18.9.2593

[24] Bachi, A., Braun, I.C., Rodrigues, J.P., Pante, N., Ribbeck, K., von Kobbe, C., Kutay, U., Wilm, M., Gorlich, D., Carmo-Fonseca, M. and Izaurralde, E. (2000) The C-terminal domain of TAP interacts with the nuclear pore complex and promotes export of specific CTE-bearing 
RNA substrates. $R N A, \mathbf{6}, 136-158$. doi:10.1017/S1355838200991994

[25] Stutz, F. and Izaurralde, E. (2003) The interplay of nuclear mRNP assembly, mRNA surveillance and export. Trends in Cell Biology, 13, 319-327. doi:10.1016/S0962-8924(03)00106-5

[26] Maruyama, K., Sato, N. and Ohta, N. (1999) Conservation of structure and cold-regulation of RNA-binding proteins in cyanobacteria: Probable convergent evolution with eukaryotic glycine-rich RNA-binding proteins. $\mathrm{Nu}$ cleic Acids Research, 27, 2029-2036. doi:10.1093/nar/27.9.2029

[27] Mans, B.J., Anantharaman, V., Aravind, L. and Koonin, E.V. (2004) Comparative genomics, evolution and origins of the nuclear envelope and pore complex. Cell Cycle, $\mathbf{3}$, 1612-1637. doi:10.4161/cc.3.12.1316

[28] Tretyakova, I., Zolotukhin, A.S., Tan, W., Bear, J., Propst, F., Ruthel, G. and Felber, B.K. (2005) NXF family protein participates in cytoplasmic mRNA trafficking. The Journal of Biological Chemistry, 280, 31981-31990. doi:10.1074/jbc.M502736200

[29] Lévesque, L., Bor, Y-C., Matzat, L.H., Jin, L., Berberoglu, S., Rekosh, D., Hammarskjöld, M.L. and Paschal, B.M. (2006) Mutations in Tap uncouple RNA export activity from translocation through the nuclear pore complex. Molecular Biology of the Cell, 17, 931-943.

[30] Katahira, J., Miki, T., Takano, K., Maruhashi, M., Uchikawa, M., Tachibana, T. and Yoneda, Y. (2008) Nuclear RNA export factor 7 is localized in processing bodies and neuronal RNA granules through interactions with shuttling hnRNPs. Nucleic Acids Research, 36, 616-628. doi:10.1093/nar/gkm556

[31] Izaurralde, E. (2001) "Friedrich Miescher Prize Awardee Lecture Review". A conserved family of nuclear export receptors mediates the exit of messenger RNA to the cytoplasm. Cellular and Molecular Life Sciences, 58, 11051112. doi:10.1007/PL00000924

[32] Lai, D., Sakkas, D. and Huang, Y. (2006) The fragile X mental retardation protein interacts with a distinct mRNA nuclear export factor NXF2. RNA, 12, 1446-1449. doi:10.1261/rna.94306

[33] Ohno, S. (1970) Evolution by Gene Duplication. SpringerVerlag, New York.

[34] Modrek, B. and Lee, C.J. (2003) Alternative splicing in the human, mouse and rat genomes is associated with an increased frequency of exon creation and/or loss. Nature Genetics, 34,177-180. doi:10.1038/ng1159

[35] Hughes, A.L. (2005) Gene duplication and the origin novel proteins. Proceedings of the National Academy of Sciences of the USA, 102, 8791-8792. doi:10.1073/pnas.0503922102

[36] Zhou, Q., Zhang, G., Zhang, Y., Xu, S., Zhao, R., Zhan, Z., Li, X., Ding, Y., Yang, S. and Wang, W. (2008) On the origin of new genes in Drosophila. Genome Research, 18, 1446-1455. doi:10.1101/gr.076588.108

[37] Brosius, J. (1991) Retroposons - Seeds of evolution. Science, 251, 753. doi:10.1126/science. 1990437

[38] Bai, Y., Casola, C. and Betran, E. (2008) Evolutionary origin of regulatory regions of retrogenes in Drosophila. BMC Genomics, 9, 241.

http://www.biomedcentral.com/1471-2164/9/241

[39] Braun, I.C., Herold, A., Rode, M., Conti, E. and Izaurralde, E. (2001) Overexpression of TAP/p15 heterodimers bypasses nuclear retention and stimulates nuclear mRNA export. The Journal of Biological Chemistry, 276, 2053620543. doi:10.1074/jbc.M100400200

[40] Erkmann, J.A. and Kutay, U. (2004) Nuclear export of mRNA: From the site of transcription to the cytoplasm. Experimental Cell Research, 296, 12-20.

[41] Cullen, B.R. (2003) Nuclear RNA export. Journal of Cell Science, 116, 587-597. doi:10.1242/jcs.00268

[42] Huang, Y.Q. and Steitz, J.A. (2005) SRprises along a messenger's journey. Molecular Cell, 17, 613-615. doi:10.1016/j.molcel.2005.02.020

[43] Björk, P. and Wieslander, L. (2011) Nucleocytoplasmic mRNP export is an integral part of mRNP biogenesis. Chromosoma, 120, 23-38. doi:10.1007/s00412-010-0298-1

[44] Strässer, K. and Hurt, E. (2000) Yralp, a conserved nuclear RNA-binding protein, interacts directly with Mex67p and is required for mRNA export. EMBO Journal, 19, 410-420. doi:10.1093/emboj/19.3.410

[45] Stutz, F., Bachi, A., Doerks, T., Braun, I.C., Séraphin, B., Wilm, M., Bork, P. and Izaurralde, E. (2000) REF, an evolutionary conserved family of hnRNP-like proteins, interacts with TAP/Mex67p and participates in mRNA nuclear export. $R N A, \mathbf{6}, 638-650$. doi: $10.1017 / \mathrm{S} 1355838200000078$

[46] Gencheva, M., Lin, T.-Y., Wu, X., Yang, L., Richard, C., Jones, M., Lin, S.-B. and Lin, R.-J. (2010) Nuclear retention of unspliced pre-mRNAs by mutant DHX16/hPRP2, a spliceosomal DEAH-box protein. The Journal of Biological Chemistry, 285, 35624-35632. doi:10.1074/jbc.M110.122309

[47] Chang, D.D. and Sharp, P.A. (1989) Regulation by HIV Rev depends upon recognition of splice sites. Cell, 59, 789-795. doi:10.1016/0092-8674(89)90602-8

[48] Legrain, P. and Rosbash, M. (1989) Some cis- and transacting mutations for splicing target pre-mRNA to the cytoplasm. Cell, 57, 573-583. doi:10.1016/0092-8674(89)90127-X

[49] Galante, P.A., Sakabe, N.J., Kirschbaum-Slager, N. and De Souza, S.J. (2004) Detection and evaluation of intron retention events in the human transcriptome. $R N A, \mathbf{1 0}$, 757-765. doi:10.1261/rna.5123504

[50] Justman, Q.A. and Clinton, G.M. (2002) Herstatin, an autoinhibitor of the human epidermal growth factor receptor 2 tyrosine kinase, modulates epidermal growth factor signaling pathways resulting in growth arrest. The Journal of Biological Chemistry, 277, 20618-20624. doi:10.1074/jbc.M111359200

[51] Forrest, S.T., Barringhaus, K.G., Perlegas, D., Hammarskjöld, M.L. and McNamara, C.A. (2004) Intron retention generates a novel Id3 isoform that inhibits vascular lesion formation. The Journal of Biological Chemistry, 279, 32897-32903. doi:10.1074/jbc.M404882200 
[52] Michael, I.P., Kurlender, L., Memari, N., Yousef, G.M., Du, D., Grass, L., Stephan, C., Jung, K. and Diamandis, E.P. (2005) Intron retention: A common splicing event within the human kallikrein gene family. Clinical Chemistry, 51, 506-515. doi:10.1373/clinchem.2004.042341

[53] Hammarskjöld, M.-L. (2001) Constitutive transport element-mediated nuclear export. Current Topics in Microbiology and Immunology, 259, 77-93. doi:10.1007/978-3-642-56597-7 4

[54] Kang, Y. and Cullen, B.R. (1999) The human Tap protein is a nuclear mRNA export factor that contains novel RNA-binding and nucleoplasmic transport sequences. Genes and Development, 13, 1126-1139. doi:10.1101/gad.13.9.1126

[55] Jin, L., Guzik, B.W., Bor, Y.-C., Rekosh, D. and Hammarskjöld, M.-L. (2003) Tap and NXT promote translation of unspliced mRNA. Genes and Development, 17, 3075-3086. doi:10.1101/gad.1155703

[56] Li, Y., Bor, Y.-C., Misawa, Y., Xue, Y., Rekosh, D. and Hammarskjöld, M.-L. (2006) An intron with a constitutive transport element is retained in a Tap messenger RNA. Nature, 443, 234-237. doi:10.1038/nature05107

[57] Ivankova, N., Tretyakova, I., Lyozin, G., Avanesyan, E., Zolotukhin, A., Zatsepina, O.G., Evgen'ev, M.B. and Mamon, L.A. (2010) Alternative transcripts expressed by small bristles, the Drosophila melanogaster $n x f 1$ gene. Gene, 458, 11-19. doi:10.1016/j.gene.2010.02.013

[58] Conti, E. and Izaurralde, E. (2005) Nonsense-mediated mRNA decay: Molecular insights and mechanistic variations cross species. Current Opinion in Cell Biology, 17, 316-325. doi:10.1016/j.ceb.2005.04.005

[59] Amrani, N., Sachs, M.S. and Jacobson, A. (2006) Early nonsense: mRNA decay solves a translational problem. Nature Reviews Molecular Cell Biology, 7, 415-425. doi:10.1038/nrm1942

[60] Bor, Y., Swartz, J., Morrison, A., Recosh, D., Ladomery, M. and Hammarskjöld, M.-L. (2006) The Wilms' tumor 1 $\left(\mathrm{WT}_{1}\right)$ gene (+KTS isoform) functions with a CTE to enhance translation from an unspliced RNA with a retained intron. Genes and Development, 20, 1597-1608. doi:10.1101/gad.1402306

[61] Coyle, J.H., Bor, Y.-C., Rekosh, D. and Hammarskjold, M.-L. (2011) The Tpr protein regulates export of mRNAs with retained introns that traffic through the $n x f 1$ pathway. $R N A, 17,1344-1356$. doi:10.1261/rna.2616111

[62] Fuke, H. and Ohno, M. (2008) Role of poly(A) tail as an identity element for mRNA nuclear export. Nucleic Acids Research, 36, 1037-1049. doi:10.1093/nar/gkm1120

[63] Mangus, D.A., Evans, M.C. and Jacobson, A. (2003) Poly(A)-binding proteins: Multifunctional scaffolds for the post-transcriptional control of gene expression. $B M C$
Genome Biology, 4, 223. doi:10.1186/gb-2003-4-7-223

[64] Behm-Ansmant, I., Gatfield, D., Rehwinkel, J., Hilgers, V. and Izaurralde, E. (2007) A conserved role for cytoplasmic poly(A)-binding protein 1 (PABPC1) in nonsense-mediated mRNA. EMBO Journal, 26, 1-11. doi:10.1038/sj.emboj.7601588

[65] Nakaya, H.I., Amaral, P.P., Louro, R., Lopes, A., Fachel, A.A., Moreira, Y.B., El-Jundi, T.A., Da Silva, A.M., Reis, E.M. and Verjovski-Almeida, S. (2007) Genome mapping and expression analyses of human intronic noncoding RNAs reveal tissue-specific patterns and enrichment in genes related to regulation of transcription. $B M C G e$ nome Biology, 8, 43.

[66] Dinger, M.E., Gascoigne, D.K. and Mattick, J.S. (2011) The evolution of RNAs with multiple functions. Biochimie, 93, 2013-2018. doi:10.1016/j.biochi.2011.07.018

[67] Wadler, C.S. and Vanderpool, C.K. (2007) A dual function for a bacterial small RNA: SgrS performs base pairing-dependent regulation and encodes a functional polypeptide. Proceeding of the National Academy of Sciences of the United States of America, 104, 20454-20459. doi:10.1073/pnas.0708102104

[68] Kageyama, Y., Kondo, T. and Hashimoto, Y. (2011) Coding vs non-coding: Translatability of short ORFs found in putative non-coding transcripts. Biochimie, 93, 19811986. doi:10.1016/j.biochi.2011.06.024

[69] Kloc, M., Foreman, V. and Reddy, S. (2011) Binary function of mRNA. Biochimie, 93, 1955-1961. doi:10.1016/j.biochi.2011.07.008

[70] Okonechnikov, K., Golosova, O. and Fursov, M. (2012) Unipro UGENE: A unified bioinformatics toolkit. Bioinformatics, 28, 1166-1167. doi:10.1093/bioinformatics/bts091

[71] Thompson, J.D., Higgins, D.G. and Gibson, T.J. (1994) CLUSTAL W: Improving the sensitivity of progressive multiple sequence alignment through sequence weighting, position-specific gap penalties and weight matrix choice. Nucleic Acids Research, 22, 4673-4680. doi:10.1093/nar/22.22.4673

[72] Notredame, C., Higgins, D.G. and Heringa, J. (2000) Tcoffee: A novel method for fast and accurate multiple sequence alignment. Journal of Molecular Biology, 302, 205-217. doi:10.1006/jmbi.2000.4042

[73] Huelsenbeck, J.P. and Ronquist, F. (2001) MRBAYES: Bayesian inference of phylogenetic trees. Bioinformatics, 17, 754-755. doi:10.1093/bioinformatics/17.8.754

[74] Markham, N.R. and Zuker, M. (2008) UNAFold: Software for nucleic acid folding and hybridization. In: Keith, J.M., Ed., Methods in Molecular Biology. Bioinformatics: Structure, Function and Applications, Humana Press, Totowa, 3-31. 